\title{
Increased GABA-A Receptor Binding and Reduced Connectivity at the Motor Cortex in Children with Hemiplegic Cerebral Palsy: A Multimodal Investigation Using ${ }^{18}$ F-Fluoroflumazenil PET, Immunohistochemistry, and MR Imaging
}

\author{
Hae-Jeong Park ${ }^{1-3}$, Chul Hoon Kim ${ }^{2-4}$, Eun Sook Park ${ }^{5}$, Bumhee Park ${ }^{1,3}$, So Ra Oh ${ }^{4}$, Maeng-Keun Oh${ }^{1}$, Chang Il Park ${ }^{5}$, \\ and Jong Doo Lee ${ }^{1-3}$ \\ ${ }^{1}$ Department of Nuclear Medicine, Yonsei University College of Medicine, Seoul, Korea; ${ }^{2}$ Severance Biomedical Science Institute, \\ Yonsei University College of Medicine, Seoul, Korea; ${ }^{3}$ Brain Korea 21 Project for Medical Science, Yonsei University College of \\ Medicine, Seoul, Korea; ${ }^{4}$ Department of Pharmacology, Yonsei University College of Medicine, Seoul, Korea; and ${ }^{5}$ Department of \\ Rehabilitation Medicine, Yonsei University College of Medicine, Seoul, Korea
}

\begin{abstract}
$\gamma$-aminobutyric acid (GABA)-A receptor-mediated neural transmission is important to promote practice-dependent plasticity after brain injury. This study investigated alterations in GABA-A receptor binding and functional and anatomic connectivity within the motor cortex in children with cerebral palsy (CP). Methods: We conducted ${ }^{18} \mathrm{~F}$-fluoroflumazenil PET on children with hemiplegic CP to investigate whether in vivo GABA-A receptor binding is altered in the ipsilateral or contralateral hemisphere of the lesion site. To evaluate changes in the GABA-A receptor subunit after prenatal brain injury, we performed GABA-A receptor immunohistochemistry using rat pups with a diffuse hypoxic ischemic insult. We also performed diffusion tensor MR imaging and resting-state functional MR imaging on the same children with hemiplegic $\mathrm{CP}$ to investigate alterations in anatomic and functional connectivity at the motor cortex with increased GABA-A receptor binding. Results: In children with hemiplegic $\mathrm{CP}$, the ${ }^{18} \mathrm{~F}$-fluoroflumazenil binding potential was increased within the ipsilateral motor cortex. GABA-A receptors with the $\alpha_{1}$ subunit were highly expressed exclusively within cortical layers III, IV, and $\mathrm{VI}$ of the motor cortex in rat pups. The motor cortex with increased GABA-A receptor binding in children with hemiplegic CP had reduced thalamocortical and corticocortical connectivity, which might be linked to increased GABA-A receptor distribution in cortical layers in rats. Conclusion: Increased expression of the GABA-A receptor $\alpha_{1}$ subunit within the ipsilateral motor cortex may be an important adaptive mechanism after prenatal brain injury in children with CP but may be associated with improper functional connectivity after birth and have adverse effects on the development of motor plasticity.
\end{abstract}

Key Words: cerebral palsy; GABA-A receptor; hemiplegic spastic cerebral palsy; connectivity

J Nucl Med 2013; 54:1263-1269

DOI: 10.2967/jnumed.112.117358

Received Nov. 21, 2012; revision accepted Mar. 18, 2013.

For correspondence contact: Jong Doo Lee, Yonsei University, College of

Medicine, 250 Seongsan-ro, Seodaemun-gu, Seoul, 120-752, Korea.

E-mail: jdlee@yuhs.ac

Published online Jun. 19, 2013.

COPYRIGHT (C) 2013 by the Society of Nuclear Medicine and Molecular Imaging, Inc.
C erebral palsy $(\mathrm{CP})$ refers to a broad range of diseases with nonprogressive permanent motor impairment that originate from insult to the immature brain. The overall prevalence of CP ranges from 1.5 to 2.5 per 1,000 children, and the number of children with $\mathrm{CP}$ has not fundamentally changed despite recent advances in prevention and therapeutic approaches (1). Among the various subtypes of $\mathrm{CP}$, the spastic type is most common, occurring in $80 \%$ of cases (2). The cause of $\mathrm{CP}$ is multifactorial, including prenatal hypoxic ischemic injury, asphyxia, infections or inflammation, coagulopathies, and inheritance $(3,4)$. Regardless of underlying etiologic factors, poor motor performance is a common manifestation in patients with $\mathrm{CP}$.

During the developmental period, the brain has a great ability to compensate for neuronal injury $(5,6)$. In particular, neural plasticity in early development allows recovery of motor performance even after hemispherectomy (7). Animal studies also show few deficits in motor function after neonatal cortical injury $(8,9)$.

Despite this great capacity for plasticity in early development, motor disability in patients with spastic CP does not significantly improve even after constraint-induced movement therapy. Patients with congenital hemiparesis, even those who have a localized infarction in the white matter region, fail to develop sufficient motor functional recovery. Therefore, there might be unknown factors that hinder development of compensatory plasticity in $\mathrm{CP}$. $\gamma$-aminobutyric acid (GABA), an inhibitory neurotransmitter, acts as a neurotropic factor, and GABA receptor-mediated neural transmission plays an important role in cortical development (10). GABAergic neurons are most vulnerable to insult at $25 \mathrm{wk}$ of gestation, a common period of premature brain injury (11). Therefore, we hypothesized that alterations in the expression of the synaptic or extrasynaptic GABA-A receptors could be a potential regulating factor in poor motor performance in patients with $\mathrm{CP}$, as neuronal excitatory-inhibitory balance is important for proper motor performance.

Previously, Lee et al. $(12,13)$, using ${ }^{18} \mathrm{~F}$-fluoroflumazenil PET, demonstrated increased regional GABA-A receptor binding potential (BP) bilaterally in the motor areas of children with spastic 
diplegic $\mathrm{CP}$ who have bilateral periventricular leukomalacia. ${ }^{18} \mathrm{~F}-$ fluoroflumazenil was recently introduced for central benzodiazepine receptor imaging $(14,15)$. However, it remains to be elucidated whether GABA-A receptor binding is increased within the ipsilateral motor cortex of the affected hemisphere or contralateral hemisphere after perinatal brain injury. It also is not yet clear which GABA-A receptor subunit among the diverse receptor subtypes is altered. Also unknown is whether the ${ }^{18} \mathrm{~F}$-fluoroflumazenil increase in the motor area is specific to spastic diplegic $\mathrm{CP}$, mostly occurring because of periventricular leukomalacia secondary to hypoxic ischemic insult, or is a common cortical adaptive process after various types of brain injury occurring during a critical period.

Therefore, we investigated ${ }^{18} \mathrm{~F}$-fluoroflumazenil BPs in children with hemiplegic $\mathrm{CP}$ who had unilateral perinatal ischemic stroke without obvious overlying cortical injury, and we performed immunohistochemistry using the brain tissues of a rat pup model with diffuse hypoxic ischemic injury to test whether the alterations in GABA-A receptor binding observed in our human imaging studies can be replicated in an animal model and to evaluate GABA-A receptor subtypes and their layer-specific distribution within the overlying cerebral cortices.

We further evaluated GABA-A receptor binding with regard to brain connectivity by means of diffusion tensor imaging (16) for anatomic connectivity and task-independent resting-state functional MR imaging for brain functional connectivity (17).

\section{MATERIALS AND METHODS}

\section{Human Subjects}

Six children with congenital hemiplegic motor impairment (spastic hemiplegic CP, 2 male and 4 female patients; age range, 7-20 y; mean $\pm \mathrm{SD}, 12.5 \pm 4.8 \mathrm{y}$ ) without a history of epilepsy were included. The underlying etiology of congenital hemiplegia was proven to be perinatal ischemic stroke on conventional MR imaging, including venous infarction or small arterial infarction involved exclusively within the white mater as described previously (18). Patients with gray matter structural abnormalities such as cortical infarction, malformation, or any other type of congenital anomaly that may have influenced the cortical biodistribution of GABA-A receptors were excluded on the basis of visual assessment of conventional MR imaging (Fig. 1). Patient demographic data, including the Gross Motor Function Classification System, are in Table 1.

Data from 18 healthy young subjects ( 9 male and 9 female) who participated in the previous ${ }^{18} \mathrm{~F}$-fluoroflumazenil PET study were used as a control group (13). Their ages ranged from 19 to $24 \mathrm{y}$, with a mean of $21.3 \pm 1.3$ y. For structural MR imaging, diffusion tensor imaging, and functional MR imaging, data from 18 age- and sex-matched healthy control subjects ( 8 male and 10 female; age range, 7-20 y; mean, $13.5 \pm 4.7$ y) from a database (13) were used. The Institutional Review Board approved this study, and all patients or their legal representatives signed a written informed consent form.

\section{F-Fluoroflumazenil PET Imaging}

The image acquisition and analysis methods for ${ }^{18} \mathrm{~F}$-fluoroflumazenil PET were the same as described in our previous paper (13). In brief, dynamic PET scans were given to 6 patients and 18 control subjects using a DSTe scanner (GE Healthcare) in a sequence of 150 frames. Voxelwise BP was calculated using a multilinear reference tissue method (19) with mean activity within the manually delineated pons as reference tissue activity using PMOD software (PMOD Technologies Ltd.). We assumed that the density of GABA-A receptors in the pons is negligible. When the brain had a left-sided

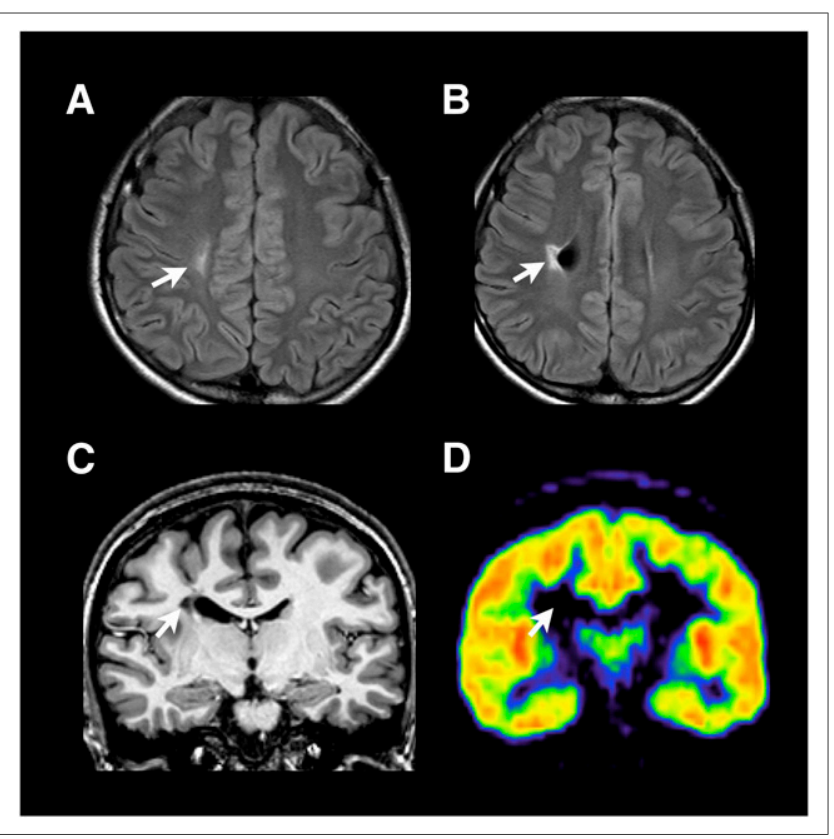

FIGURE 1. A 20-y-old woman with left hemiplegia. (A and B) Fluidattenuated inversion recovery MR axial images show focal ischemic infarction within right centrum semiovale with focal dilatation of lateral ventricle. (C) T1-weighted coronal image shows periventricular focal infarction extending to corticospinal tract without overlying gray matter injury. These findings are compatible with focal venous infarction. (D) Fluoroflumazenil binding-potential map corresponding to slice in $\mathrm{C}$ shows increased BP at affected-side motor cortices. Arrows indicate lesion site.

lesion, we flipped brain images to locate the lesion side to the right hemisphere.

Spatial preprocessing and statistical analysis of BP maps were performed using SPM8 software (Institute of Neurology, University College London). A BP map of each individual was transformed to the group template in Montreal Neurologic Institute (MNI) space using a nonlinear transform function of the individual T1-weighted image in BP space to the template. These maps were convolved with an isotropic gaussian kernel of $8 \mathrm{~mm}$ in full width at half maximum. To evaluate regional alterations in BP, we remove the effects of global differences in BP by scaling the BP of each voxel with the mean BP from the entire brain. After voxelwise application of a 2-sample $t$ test between groups, clusters composed of a minimum of 65 contiguous voxels with a threshold of $P<0.001$ (uncorrected) were considered significant, corresponding to $P<0.05$ corrected for multiple comparisons estimated by $10,000 \times$ Monte Carlo simulations (20).

We also compared the ${ }^{18} \mathrm{~F}$-fluoroflumazenil BP in the affected and intact sides to compensate for age effects in the group comparison study (supplemental data, available online at http://jnm.snmjournals.org).

\section{Animal Hypoxic Model}

Five rat pups ( $7 \mathrm{~d}$ old, equivalent to $23-36 \mathrm{wk}$ of gestation in humans (21)) were subjected to $2 \mathrm{~h}$ of hypoxia in a humidified hypoxia chamber with ambient $\mathrm{O}_{2}$ levels continuously regulated at around 5\%. We performed these experiments in accordance with the requirements of the Institutional Animal Care and Use Committee of Yonsei University College of Medicine. The chamber was flushed with $95 \% \mathrm{~N}_{2}$ and $5 \% \mathrm{CO}_{2}$ for $2 \mathrm{~h}$ to keep the rats under hypoxic conditions. Hypoxia was chosen as an insult because prenatal hypoxia is implicated in most cases of CP. Control rats were kept under normoxic conditions. The ambient temperature of the chamber was controlled at $37.5^{\circ} \mathrm{C}$ by 
TABLE 1

Demographic Data of Patients with Hemiplegic CP

\begin{tabular}{|c|c|c|c|c|c|c|c|}
\hline Patient no. & Sex & Age (y) & Lesion side & MR imaging diagnosis & Birth weight (kg) & Delivery (mo) & GMFCS \\
\hline 1 & $\mathrm{~F}$ & 20 & L & Focal infarction (corona radiata) & 3 & 40 & 1 \\
\hline 2 & $\mathrm{~F}$ & 10 & $\mathrm{R}$ & Focal infarction (corona radiata) & 4 & 40 & 1 \\
\hline 3 & M & 15 & $\mathrm{R}$ & Focal infarction (corona radiata) & 4.1 & 42 & 1 \\
\hline 4 & M & 7 & $\mathrm{~L}$ & $\begin{array}{l}\text { Focal infarction (white matter and } \\
\text { basal ganglia) }\end{array}$ & 3.4 & 40 & 1 \\
\hline 5 & $\mathrm{~F}$ & 14 & L & Focal infarction (internal capsule) & 2.3 & 40 & 1 \\
\hline 6 & $\mathrm{~F}$ & 9 & $\mathrm{R}$ & Periventricular leukomalacia (left $>$ right) & 1.6 & 31 & 2 \\
\hline
\end{tabular}

water circulation from a temperature-controlled bath. This hypoxic ischemia method was chosen as an animal model of CP because this method preserves the cortices relatively well without severe cerebral ischemia, which is generally entailed in the model of middle cerebral artery occlusion. Among fluoroflumazenil binding sites, $\alpha_{1}, \alpha_{2}$, and $\alpha_{3}$ subunits are critical during development; we examined expression patterns of these 3 subunits using immunohistochemistry.

\section{GABA-A Receptor Immunohistochemistry in Animal Model}

Rat pups that were kept under normoxic conditions $(n=5)$ or exposed to hypoxia $(n=5)$ at postnatal day 7 were anesthetized by an intraperitoneal injection of ketamine and xylazine and perfused intracardially with $4 \%$ paraformaldehyde in phosphate-buffered saline at $10 \mathrm{~d}$ after birth (corresponding to $36-40 \mathrm{wk}$ in humans). The brains were removed and fixed in $4 \%$ paraformaldehyde for $24 \mathrm{~h}$ at $4{ }^{\circ} \mathrm{C}$ and then were immersed in $30 \%$ sucrose in phosphate-buffered saline until they sank. They then were cryosectioned into $40-\mu \mathrm{m}$ sagittal sections (1.2-1.4 mm lateral to the midline) in a cryostat. After permeabilization with $0.2 \%$ Triton X-100 (The Dow Chemical Co.), sections were incubated with $3 \%$ bovine serum albumin in phosphate-buffered saline for $1 \mathrm{~h}$.

Rabbit polyclonal GABA-A receptor $\alpha_{1}$ subunit antibody (Alomone), $\alpha_{2}$ subunit antibody (Sigma), and $\alpha_{3}$ subunit antibody (Alomone) were applied at $1: 250$ at $4^{\circ} \mathrm{C}$ overnight followed by horseradish peroxidase-conjugated antirabbit secondary antibody at 1:250 for $2 \mathrm{~h}$. After being washed 3 times, the sections were treated with diaminobenzidine tetrahydrochloride and $\mathrm{H}_{2} \mathrm{O}_{2}$. Brain sections were placed on glass slides, mounted using Permount medium (Fisher Scientific), and qualitatively analyzed by an experienced specialist. Densitometric analysis was then performed to confirm the visual evaluation results.

\section{Tract-Based Spatial Statistics of the White Matter Anisotropy in Patients with Hemiplegic CP}

All structural MR imaging, diffusion tensor imaging, and restingstate functional MR imaging were performed using a 3.0-T scanner (Intera; Philips Medical Systems) and analyzed using the same method as described in our previous paper (13). In brief, high-resolution T1-weighted MR imaging volumes were acquired with a $0.859 \times$ $0.859 \times 1.2 \mathrm{~mm}$ voxel size. We performed diffusion tensor imaging using a single-shot echo-planar acquisition from 45 diffusion-encoded gradient directions with $1.72 \times 1.72 \times 2 \mathrm{~mm}$ voxels, an echo time of $60 \mathrm{~ms}$, and a b-factor of $600 \mathrm{~s} / \mathrm{mm}^{2}$.

We flipped all MR images to locate the lesion in the right hemisphere when the brain had a left-sided lesion. The fractional anisotropy (FA) values were analyzed using a tract-based spatial statistics technique (22) including eddy current correction, generation of a mean FA skeleton, projection of each subject's FA map onto the mean FA skeleton, and voxelwise statistical evaluation of FA in the skeleton. To correct for multiple comparisons using the threshold-free cluster enhancement approach in tract-based spatial statistics, regional FA differences were given a threshold at the level of threshold-free cluster enhancement-corrected $P<0.05$. We also evaluated the hemispheric difference in FA in patients by comparing initial FA data used for the group comparison and flipped data using the method described above.

\section{Statistical Analysis of Resting-State Motor Functional Connectivity in Patients with Hemiplegic CP}

We acquired 165 functional MR imaging scans from each subject using an echo-planar sequence with a voxel size of $2.75 \times 2.75 \times 4.8 \mathrm{~mm}$, a repetition time of $2,000 \mathrm{~ms}$, and an echo time of $30 \mathrm{~ms}$. During functional MR imaging, the subjects were instructed to stay awake with their eyes closed, without focusing on a specific thought and without moving. After removal of the first 5 scans, the remaining 160 scans underwent acquisition time delay correction and head motion correction, followed by spatial normalization by application of nonlinear transformation from the coregistered T1-weighted image in the functional MR imaging space to the MNI template using SPM8. Motion effects were further corrected using independent component analysis by reducing components highly correlated $(>0.7)$ with 1 of 6 motion parameters. Finally, normalized data were spatially smoothed using a gaussian filter of $4 \mathrm{~mm}$ in full width at half maximum.

To evaluate functional connectivity, correlation coefficients between the time series of the ${ }^{18} \mathrm{~F}$-fluoroflumazenil-increased region in the primary motor cortex in $\mathrm{CP}$ and those of entire brain voxels were derived after band-pass filtering $(0.01-0.08 \mathrm{~Hz})$ and regressing out motion effects and global signal changes at white matter, cerebrospinal fluid, and whole brain. To examine group differences, a 2-sample $t$ test was applied to $z$ values of the correlation coefficients after the Fisher $r$-to- $z$ transformation for each voxel. Clusters with a minimum of 39 contiguous voxels with a threshold of $P<0.005$ (uncorrected) were considered significant, equivalent to $P<0.05$ corrected by the cluster size threshold as described above.

\section{RESULTS}

Statistical parametric maps of intergroup differences in ${ }^{18} \mathrm{~F}-\mathrm{flu}$ oroflumazenil BP revealed that, in comparison with the control group, the patients with hemispheric CT had increased GABA-A receptor $\mathrm{BP}$ in the primary motor cortices (paracentral gyrus and precentral gyrus) and the occipital cortex of the affected hemisphere (Fig. 2; Table 2). When we compared the ${ }^{18} \mathrm{~F}$-fluoroflumazenil BP of the affected hemisphere with that of the intact hemisphere, a similar increase was found in the affected side specifically in the paracentral gyrus, precentral gyrus, anterior cingulate gyrus, and postcentral gyrus (Supplemental Fig. 1). 


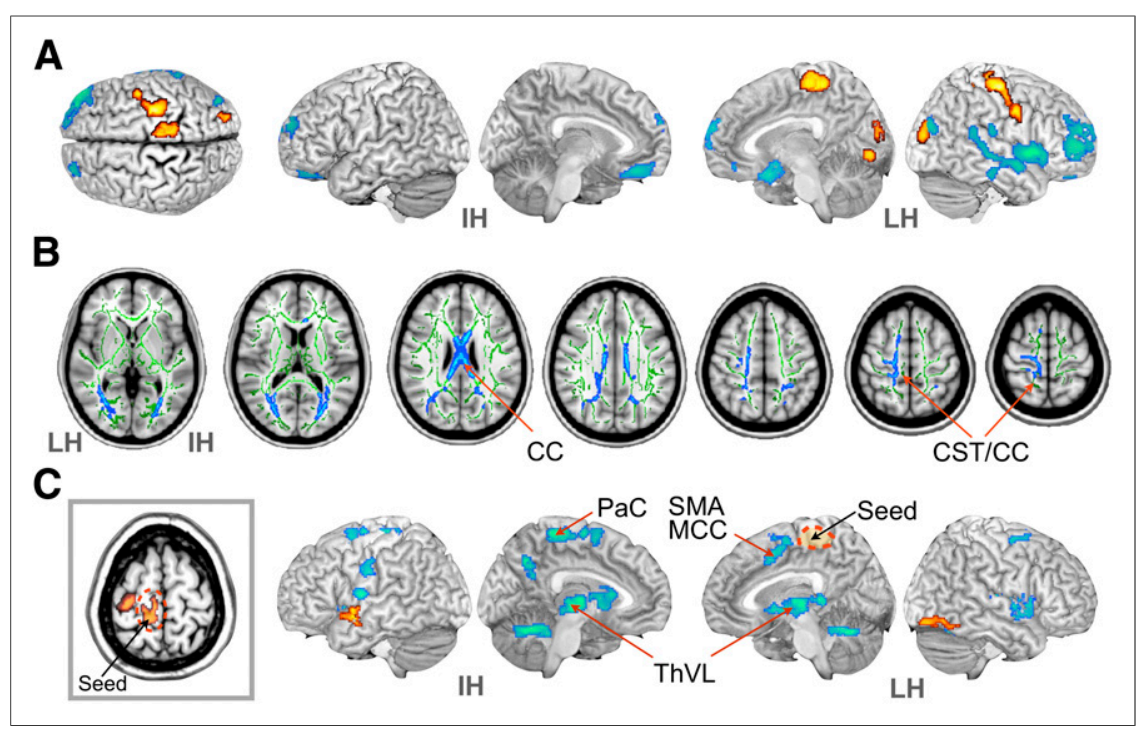

FIGURE 2. Statistical maps of fluoroflumazenil binding potential, diffusion anisotropy, and functional connectivity in patients with hemiplegic CP. (A) Map of regional differences in fluoroflumazenil binding potential. Red indicates increased binding potential in patients with hemiplegic $\mathrm{CP}$, compared with healthy controls; blue indicates reduced binding potential in hemiplegic CP, compared with controls $(P<0.05$, corrected cluster size). (B) Regions of decreased FA in hemiplegic CP, compared with controls $(P<0.005$, threshold-free cluster enhancement-corrected [blue] on white matter skeleton [green]). (C) Group difference in functional connectivity between controls and hemiplegic CP from seed region detected using 2-sample $t$ test $(P<0.05$, corrected cluster size). A seed region (dashed circle) was chosen in paracentral gyrus of affected hemisphere (rectangle), where hemiplegic CP had increased fluoroflumazenil binding potential. Red indicates increased connectivity from seed region in hemiplegic CP; blue indicates reduced connectivity from seed region, compared with controls. CC = corpus callosum; CST = corticospinal tract; $\mathrm{IH}=$ intact hemisphere; $\mathrm{LH}=$ lesioned hemisphere; $\mathrm{MCC}=$ middle cingulate gyrus; $\mathrm{PaC}=$ paracentral lobule; $\mathrm{SMA}=$ supplementary motor area; ThVL = ventrolateral thalamus.

GABA-A receptor $\alpha_{1}$ immunoreactivity increased exclusively in motor areas in rats exposed to hypoxic conditions, compared with wild-type normal controls (Fig. 3A, top). Preincubation of GABA-A receptor $\alpha_{1}$ subunit antibody with an $\alpha_{1}$ blocking peptide blocked $\alpha_{1}$ immunoreactivity in cortical slices (Fig. 3A, bottom). $\alpha 1$ immunoreactivity increased selectively in cortical layers III, IV, and VI (Fig. 3B). In contrast, the expression of GABA-A receptor $\alpha_{2}$ and $\alpha_{3}$ subunits was not significantly altered in this study (Fig. 3C).

Tract-based spatial statistical analysis showed reduced FA over a wide area, primarily in the affected hemisphere. FA reduction within patients with hemispheric $\mathrm{CP}$, compared with controls, was observed in the white matter around the motor area and corpus callosal fibers (Fig. 2B). Compared with the contralateral hemisphere, the lesion side showed similarly reduced anisotropy in the thalamus, corpus callosum, and corona radiata (Supplemental Fig. 2).

The paracentral gyrus with increased ${ }^{18} \mathrm{~F}$-fluoroflumazenil in the affected hemisphere of patients with $\mathrm{CP}$ had reduced functional connectivity with the motor-related regions such as the ipsilateral middle cingulate gyrus, supplementary motor area, right and left thalamus, and contralateral motor areas (Fig. 2C).

As summarized in Figure 4, the increased ${ }^{18} \mathrm{~F}$-fluoroflumazenil $\mathrm{BP}$ regions in the paracentral gyrus of the affected hemisphere had reduced functional connectivity with the bilateral thalamus and motor cortical area. The decreased FA area in patients with hemi- plegic $\mathrm{CP}$ corresponded to the functional connectivity results (Fig. 4A). This altered connectivity in neuroimaging data was highly associated with neural circuits corresponding to cortical layers III, IV, and VI, where overexpressed GABAA receptor $\alpha_{1}$ subunit was observed in rats (Fig. 4B).

\section{DISCUSSION}

In this study, patients with hemiplegic CP showed increased ${ }^{18} \mathrm{~F}$-fluoroflumazenil $\mathrm{BP}$ within the affected-side motor cortex. Since ${ }^{18} \mathrm{~F}$-fluoroflumazenil PET is not specific for GABA receptor subunit types and does not resolve their distributions within cortical layers, this study using immunohistochemistry of rat pups revealed that the GABA-A receptors were hyperpolarizing the $\alpha_{1}$ subunit and were highly expressed exclusively within cortical layers III, IV, and VI of the motor cortex in the rat model. The motor cortex of patients with increased GABA-A receptor binding had reduced functional and anatomic connectivity with the thalamus or other cortical regions, as might be linked to increased GABA-A receptor distribution in cortical layers in rats.

In addition to the increased ${ }^{18} \mathrm{~F}$-fluoroflumazenil $\mathrm{BP}$ at the motor cortex, increased ${ }^{18} \mathrm{~F}$-fluoroflumazenil $\mathrm{BP}$ at the visual cortex and decreased $\mathrm{BP}$ at the frontal lobe were similarly found and discussed in our previous studies with diplegic CP $(12,13)$. The frontal lobe decrease of ${ }^{18} \mathrm{~F}$-fluoroflumazenil BP was also evidenced in a postmortem study that showed a significant loss of GABAergic neurons and receptor density at the frontal regions of infants with periventricular leukomalacia (23).

Since the underlying etiologies of hemiplegic spastic CP are different from those of diplegic spastic $\mathrm{CP}$, the consistent ${ }^{18} \mathrm{~F}$ fluoroflumazenil BP finding between patients with hemiplegic and diplegic CP (13) suggests that the ${ }^{18} \mathrm{~F}$-fluoroflumazenil BP increase in the motor cortex may be a common effect of neural processes after various types of brain injury during a critical period. The current study with hemiplegic $\mathrm{CP}$ provides further evidence that the ${ }^{18} \mathrm{~F}$-fluoroflumazenil BP increase was in the affected-side motor cortex rather than the contralateral hemisphere. Considering a well-known phenomenon that the intact contralateral hemisphere is often reorganized to compensate symptomatic deficits caused by the lesion, we may not simply associate the ipsilateral ${ }^{18} \mathrm{~F}$-fluoroflumazenil BP alteration with the compensatory plasticity.

The increased GABA-A BP at the motor cortex in the older children with $\mathrm{CP}$ (mean age, $12.5 \mathrm{y}$ ) was replicated in the 10-d-old hypoxic rat pups (corresponding to $36-40$ wk of gestation in humans (21)). This replication in very young brains implies that increased GABA-A receptor binding in the motor cortex may be associated with adaptive processes after brain damage during prenatal development, rather than practice-dependent changes (i.e., compensatory plasticity) during childhood. 
TABLE 2

Results of Regional ${ }^{18}$ F-Fluoroflumazenil Binding-Potential Analysis

\begin{tabular}{|c|c|c|c|c|c|}
\hline Contrast & Region & Brodmann area & MNI $x, y, z(\mathrm{~mm})$ & Zmax & Cluster size \\
\hline \multirow[t]{7}{*}{$\mathrm{C}<\mathrm{HCP}$} & R precentral gyrus & 4 & $30,-20,58$ & 4.68 & 254 \\
\hline & $\mathrm{R}$ middle frontal gyrus & 6 & $36,-12,48$ & 3.68 & - \\
\hline & R precentral gyrus & 6 & $46,-4,36$ & 4.15 & 67 \\
\hline & $\mathrm{R}$ paracentral gyrus & 6 & $10,-30,58$ & 4.1 & 244 \\
\hline & R middle occipital gyrus & 18 & $24,-88,16$ & 3.81 & 111 \\
\hline & R precuneus & 31 & $26,-82,24$ & 3.58 & - \\
\hline & $\mathrm{R}$ lingual gyrus & 18 & $14,-80,-2$ & 3.73 & 71 \\
\hline \multirow[t]{8}{*}{$\mathrm{HCP}<\mathrm{C}$} & R superior temporal gyrus & 22 & $54,8,-2$ & 5.28 & 797 \\
\hline & R superior/inferior frontal gyrus & 10 & $38,56,18$ & 4.6 & 934 \\
\hline & $\mathrm{R}$ middle temporal gyrus & 21 & $62,-4,-12$ & 4.35 & 500 \\
\hline & R superior occipital gyrus & 19 & $40,-82,24$ & 4.17 & 89 \\
\hline & $\mathrm{R}$ inferior frontal gyrus & 47 & $22,12,-22$ & 4.13 & 334 \\
\hline & L medial/inferior frontal gyrus & 11 & $-4,38,-18$ & 4.05 & 233 \\
\hline & $\mathrm{L}$ middle frontal gyrus & 10 & $-26,58,24$ & 3.95 & 126 \\
\hline & R postcentral gyrus & 40 & $58,-26,18$ & 3.84 & 69 \\
\hline
\end{tabular}

Data are regions with fluoroflumazenil binding-potential differences between healthy controls and patients with hemiplegic CP. $P<0.05$ corrected by cluster level threshold, which is equivalent to $P<0.001$ uncorrected, cluster size $>65$. $\mathrm{C}=$ healthy controls; $\mathrm{HCP}=$ patients with hemiplegic $\mathrm{CP} ; \mathrm{L}=$ intact hemisphere; $\mathrm{MNI} x, y, z=$ Montreal Neurological Institute coordinates: $\mathrm{R}=$ affected hemisphere; $Z \max =z$ maximum within cluster.

The immunohistochemistry of hypoxic rat pups further suggests that the increased ${ }^{18} \mathrm{~F}$-fluoroflumazenil BP in the motor cortices of the human brain may be attributable to hyperpolarizing $\alpha_{1}$ subunit-type GABA-A receptors, under the plausible assumption of no additional alteration in the GABA-A subunit after prenatal development. The underlying developmental mechanism of the increased GABA-A receptor $\alpha_{1}$ subunits in the affected-side motor cortical areas of spastic CP is not clear. To explain CP etiology, early damage of the subplate neurons is often supposed. Ablation of the subplate neuron in the visual ocular column inhibited normal developmental changes in GABA-A receptors and increased $\alpha_{2}$ and $\alpha_{3}$ subunits without conversion to $\alpha_{1}$ subunits (24). However, the increased $\alpha_{1}$ subunits found in this study might not be explained by the traditional subplate neuronal injury theory, which has focused mainly on the development of the sensory cortex via thalamocortical circuits (24). Thus, mechanisms regulating expression of GABA-A receptor $\alpha_{1}$ subunits in the motor area remain to be elucidated.

This overexpressed pattern of GABA-A receptor $\alpha_{1}$ subunit due to brain injury during the developmental period contradicts the expression pattern after brain injury in adults, in whom GABA-A receptor expression has to be reduced to facilitate compensatory plasticity (25-27). This contradiction of GABA-A receptor expression in early and adult brain damage is not surprising since the motor cortex of congenital hemiplegia patients has shown increased metabolism (28) whereas decreased glucose metabolism has been shown in adults damaged by stroke (29). It is well known that cortical disinhibition promotes neural plasticity, especially practice-dependent plasticity (30). Ischemic conditions in the mature brain cause transient downregulation of GABA-A receptors in the affected periinfarct viable neurons or contralateral hemisphere because of extracellular liberation of excessive GABA, thereby prompting practicedependent plasticity in the motor cortex (31). Conversely, long-term increases in GABA-A receptor function in the motor cortex prevent development of proper practice-dependent plasticity (30) or block somatosensory input-driven motor excitability in the adult brain (32). Therefore, the increased GABA-A receptor binding might adversely affect compensatory plasticity in patients with $\mathrm{CP}$ by preventing effective reorganizations between motor areas for the produc-

\author{
tion of movement.
}

FIGURE 3. Hypoxia-increased expression of GABA-A receptor $\alpha_{1}$ subunit in rat motor cortex. (A, top) Immunohistochemistry was performed using GABA-A $\alpha_{1}$ receptor subunit antibody in sagittal sections of rat brains removed from 10-d-old rat pups preexposed to $5 \%$ hypoxia for $2 \mathrm{~h}$ at $7 \mathrm{~d}$ after birth. (A, bottom) Blocking peptide for $\alpha_{1}$ receptor subunit antibody was used to show specificity of $\alpha_{1}$ immunosignals. (B) Motor cortical layers were defined using Nissl stain and GABA-A $\alpha_{1}$ receptor immunohistochemistry. (C) Immunohistochemical analysis was performed as in A for GABA-A $\alpha_{2}$ and $\alpha_{3}$ receptor subunits. 


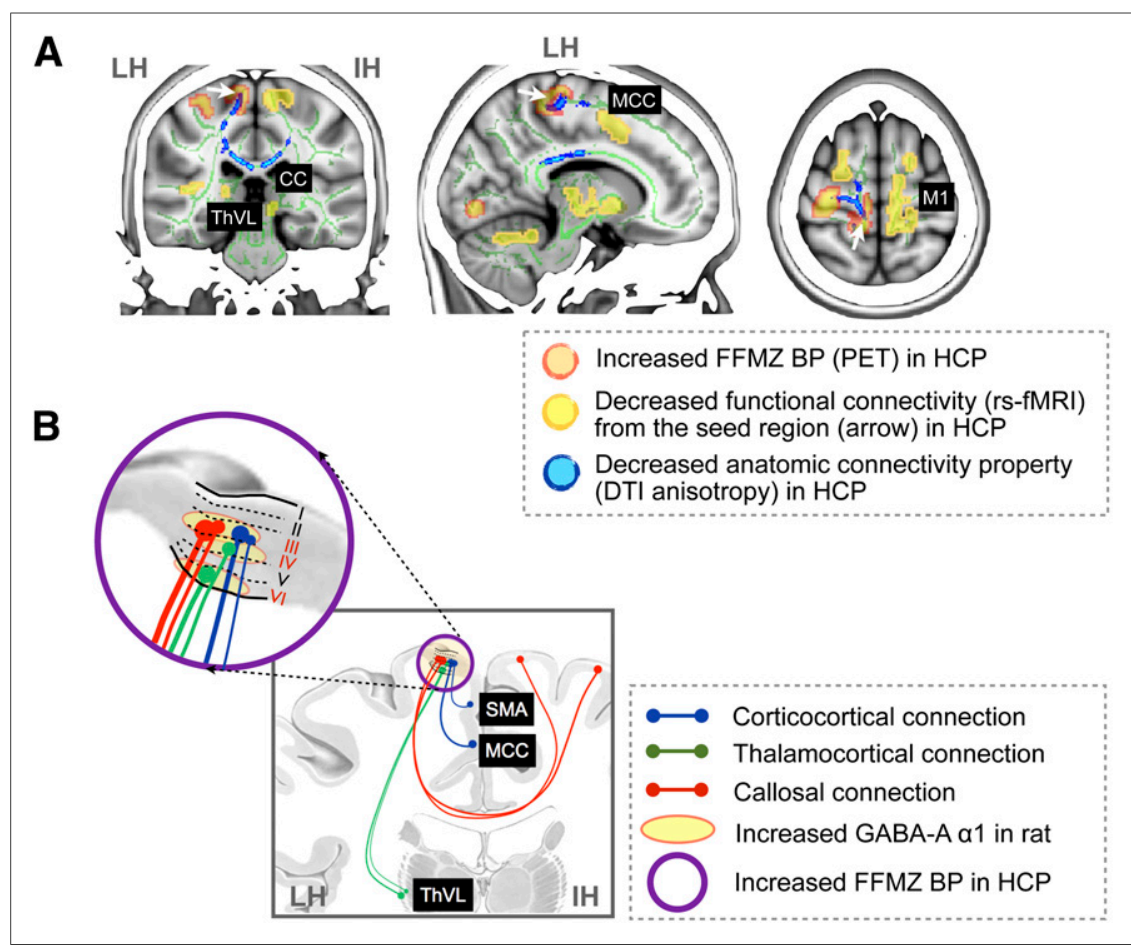

FIGURE 4. Summary of neuroimaging results and animal study results. (A) Fluoroflumazenil BP-increased regions (red in Fig. 3A), diffusion anisotropy-decreased regions (blue in Fig. $3 \mathrm{~B}$ ), and functional connectivity-increased regions (yellow in Fig. $3 \mathrm{C}$ ) in patients with hemiplegic CP were overlaid on MR coronal, sagittal, and axial sections. Both functional connectivity from increased fluoroflumazenil BP region defined using resting-state functional MR imaging and corresponding anatomic connectivity property defined by FA of diffusion tensor imaging were reduced in hemiplegic CP. (B) Summary illustration shows motor cortex with reduced connectivity in patients with hemiplegic CP (Fig. 3) and neural circuits corresponding to cortical layers III, IV, and VI with overexpressed GABA-A $\alpha_{1}$ in rats (Fig. 2). CC = corpus callosum; DTI = diffusion tensor imaging; FFMZ = fluoroflumazenil; HCP = hemiplegic $\mathrm{CP} ; \mathrm{IH}=$ intact hemisphere; $\mathrm{LH}=$ lesioned hemisphere; $\mathrm{M} 1$ = primary motor cortex; $\mathrm{MCC}=$ middle cingulate gyrus; rs-fMRI = resting-state functional MR imaging; SMA = supplementary motor area; $\mathrm{ThVL}=$ ventrolateral thalamus.

In the current study, overexpression of the inhibitory GABA-A receptor $\alpha_{1}$ subunit was observed mainly in layers III, IV, and VI of the rat motor cortex. Because cortical layer III is the origin and target of the intracortical and commissural connections, whereas layers IV and VI are the main sites of interconnection between the cortex and the thalamus, increased inhibitory neuronal receptors in these layers of the rat brain may be associated with the decrease in corticocortical, thalamocortical, and interhemispheric connections as revealed on our human neuroimaging data (Fig. 4).

Overexpression of GABA-A receptors in rat cortical layer III may be linked to reduced anisotropy in the callosal fibers and fibers linking motor areas in the affected hemisphere of patients with hemiplegic CP since these fibers are anchored mainly in cortical layer III. Abnormally low diffusion anisotropy in the anterior part of the isthmus of the corpus callosum, a major pathway for callosal fibers of the motor cortex (33), may be well associated with decreased functional interhemispheric connectivity between contralateral motor areas of patients in resting-state functional MR imaging (Fig. 4A).

Within the affected hemisphere, motor areas with increased ${ }^{18} \mathrm{~F}$ fluoroflumazenil binding in patients with spastic hemiplegic $\mathrm{CP}$ showed reduced functional connectivity with the ipsilateral cingulate motor area and supplementary motor area. Considering that the cingulate motor area $(34,35)$ and supplementary motor area (36) are involved in higher-order control of movements, the inhibitory neurons in layer III of the motor cortex might hinder appropriate input from these areas for motor performance. The reduced functional connectivity between the motor cortex and the right and left ventrolateral thalamus and reduced diffusion anisotropy in the lesion-side thalamic white matter could be linked to the increased GABA-A receptor expression in layers VI and IV, which are main targets for thalamocortical circuits.

Of interest, the regions showing increased GABA-A receptor binding within the overlying gray matter are linked well to the regions of reduced diffusion anisotropy within the white matter, as might be related to the reduced intra- and interhemispheric functional connectivity (Fig. 4A). In fact, restoration of the interhemispheric and intrahemispheric connection after brain injury is important for compensation of motor deficits $(37,38)$. However, children with hemiplegic $\mathrm{CP}$ showed permanent reduced connections, which might be one of the important mechanisms of permanent poor motor function in $\mathrm{CP}$ patients.

As a limitation of the current study with hemiplegic patients, the fact that data were obtained from only 6 patients according to the selection criteria of morphologic intactness in the cortex could result in relatively low statistical power for group comparisons between controls. Age differences exist between groups for the ${ }^{18} \mathrm{~F}$ fluoroflumazenil analysis since we could not acquire ${ }^{18} \mathrm{~F}$-fluoroflumazenil PET data from age-matched children because of radiation hazards. Considering that the flumazenil volume of distribution declines as a function of age (39), we applied the global intensity normalization of BP to examine regional differences. We also examined hemispheric differences to compensate for age differences. Although hemispheric asymmetry might exist in the young controls, we found increased ${ }^{18} \mathrm{~F}$-fluoroflumazenil BPs specifically in the motor cortex of the affected side of patients with hemiplegic CP.

The interindividual variability of motor symptoms in the patient group was too low (mostly, Gross Motor Function Classification System level 1 as shown in Table 1) to reveal relationships between findings from ${ }^{18} \mathrm{~F}$-fluoroflumazenil BPs and FAs in diffusion tensor imaging or functional connectivity in resting-state functional MR imaging. In the preliminary correlation analyses for the current results (not presented in this paper), we found a tendency toward a negative correlation between ${ }^{18} \mathrm{~F}$-fluoroflumazenil BPs at the paracentral gyrus and FAs at the corpus callosum in the affected hemisphere $(r=-0.811 ; P=0.05)$. However, we did not detect any significant correlations between ${ }^{18} \mathrm{~F}$-fluoroflumazenil $\mathrm{BPs}$ and thalamocortical functional connectivity at the lesion-side motor cortex. This finding was not surprising since there was no significant thalamocortical functional connectivity at the lesioned 
hemisphere when we applied 1-sample $t$ testing for this functional connectivity.

Because of the small sample size with relatively uniform clinical symptoms, we did not correlate current findings with symptoms. When a sufficient number of datasets with diverse symptom levels is accumulated, the findings of the current study would be significantly extended by associating neuroimaging data with behavioral and neurophysiologic data such as motor symptoms and GABA-A-mediated intracortical inhibition.

\section{CONCLUSION}

A GABA-A receptor binding study using ${ }^{18} \mathrm{~F}$-fluoroflumazenil PET and a connectivity study using MR imaging techniques clearly demonstrated that alterations in the affected motor cortical GABA-A receptor binding could be an important underlying pathophysiologic mechanism of motor dysfunction in $\mathrm{CP}$ patients.

\section{DISCLOSURE}

The costs of publication of this article were defrayed in part by the payment of page charges. Therefore, and solely to indicate this fact, this article is hereby marked "advertisement" in accordance with 18 USC section 1734. This work was supported by National Research Foundation of Korea (NRF) grant 20100020676 funded by the Korean government (MEST) and by a 2007 faculty research grant (6-2007-0109) from Yonsei University College of Medicine. No other potential conflict of interest relevant to this article was reported.

\section{REFERENCES}

1. Kuban KC, Leviton A. Cerebral palsy. N Engl J Med. 1994;330:188-195.

2. Stanley F, Blair E, Alberman E. Cerebral Palsies: Epidemiology and Causal Pathways. London, U.K.: Mac Keith Press; 2000:14.

3. Msall ME. Developmental vulnerability and resilience in extremely preterm infants. JAMA. 2004;292:2399-2401.

4. Keogh JM, Badawi N. The origins of cerebral palsy. Curr Opin Neurol. 2006;19:129-134.

5. Staudt M. (Re-)organization of the developing human brain following periventricular white matter lesions. Neurosci Biobehav Rev. 2007;31:1150-1156.

6. Juenger H, Grodd W, Krageloh-Mann I, Staudt M. (Re-)organization of basal ganglia in congenital hemiparesis with ipsilateral cortico-spinal projections. $\mathrm{Neu}$ ropediatrics. 2008;39:252-258.

7. Kossoff EH, Vining EP, Pillas DJ, et al. Hemispherectomy for intractable unihemispheric epilepsy etiology vs outcome. Neurology. 2003;61:887-890.

8. Rouiller EM, Yu XH, Moret V, Tempini A, Wiesendanger M, Liang F. Dexterity in adult monkeys following early lesion of the motor cortical hand area: the role of cortex adjacent to the lesion. Eur J Neurosci. 1998;10:729-740.

9. Kolb B, Whishaw IQ. Plasticity in the neocortex: mechanisms underlying recovery from early brain damage. Prog Neurobiol. 1989;32:235-276.

10. Represa A, Ben-Ari Y. Trophic actions of GABA on neuronal development. Trends Neurosci. 2005;28:278-283.

11. Das SK, Ray PK. Ontogeny of GABA pathway in human fetal brains. Biochem Biophys Res Commun. 1996;228:544-548.

12. Lee JD, Park HJ, Park ES, et al. Assessment of regional GABA(A) receptor binding using ${ }^{18} \mathrm{~F}$-fluoroflumazenil positron emission tomography in spastic type cerebral palsy. Neuroimage. 2007;34:19-25.

13. Lee JD, Park HJ, Park ES, et al. Motor pathway injury in patients with periventricular leucomalacia and spastic diplegia. Brain. 2011;134:1199-1210.

14. Mitterhauser M, Wadsak W, Wabnegger L, et al. Biological evaluation of $2^{\prime}-\left[{ }^{18} \mathrm{~F}\right]$ fluoroflumazenil ([ $\left.\left.{ }^{18} \mathrm{~F}\right] \mathrm{FFMZ}\right)$, a potential GABA receptor ligand for PET. Nucl Med Biol. 2004;31:291-295.
15. Chang YS, Jeong JM, Yoon YH, et al. Biological properties of $2^{\prime}$ - $\left[{ }^{18} \mathrm{~F}\right]$ fluoroflumazenil for central benzodiazepine receptor imaging. Nucl Med Biol. 2005;32: 263-268.

16. Basser PJ, Mattiello J, LeBihan D. MR diffusion tensor spectroscopy and imaging. Biophys J. 1994;66:259-267.

17. Biswal B, Yetkin FZ, Haughton VM, Hyde JS. Functional connectivity in the motor cortex of resting human brain using echo-planar MR imaging. Magn Reson Med. 1995;34:537-541.

18. Kirton A, Deveber G, Pontigon AM, Macgregor D, Shroff M. Presumed perinatal ischemic stroke: vascular classification predicts outcomes. Ann Neurol. 2008;63: 436-443.

19. Ichise M, Ballinger JR, Golan H, et al. Noninvasive quantification of dopamine D2 receptors with iodine-123-IBF SPECT. J Nucl Med. 1996;37:513-520.

20. Xiong J, Gao JH, Lancaster JL, Fox PT. Clustered pixels analysis for functional MR imaging activation studies of the human brain. Hum Brain Mapp. 1995;3: 287-301.

21. Hagberg H, Peebles D, Mallard C. Models of white matter injury: comparison of infectious, hypoxic-ischemic, and excitotoxic insults. Ment Retard Dev Disabil Res Rev. 2002;8:30-38.

22. Smith SM, Jenkinson M, Johansen-Berg H, et al. Tract-based spatial statistics: voxelwise analysis of multi-subject diffusion data. Neuroimage. 2006;31:14871505 .

23. Robinson S, Li Q, Dechant A, Cohen ML. Neonatal loss of gamma-aminobutyric acid pathway expression after human perinatal brain injury. J Neurosurg. 2006; 104:396-408.

24. Kanold PO, Luhmann HJ. The subplate and early cortical circuits. Annu Rev Neurosci. 2010;33:23-48.

25. Yamauchi H, Kudoh T, Kishibe Y, Iwasaki J, Kagawa S. Selective neuronal damage and chronic hemodynamic cerebral ischemia. Ann Neurol. 2007;61:454-465.

26. Redecker C, Wang W, Fritschy JM, Witte OW. Widespread and long-lasting alterations in GABA(A)-receptor subtypes after focal cortical infarcts in rats: mediation by NMDA-dependent processes. J Cereb Blood Flow Metab. 2002; 22:1463-1475.

27. Clarkson AN, Huang BS, Macisaac SE, Mody I, Carmichael ST. Reducing excessive GABA-mediated tonic inhibition promotes functional recovery after stroke. Nature. 2010;468:305-309.

28. Vandermeeren Y, Olivier E, Sebire G, et al. Increased FDG uptake in the ipsilesional sensorimotor cortex in congenital hemiplegia. Neuroimage. 2002;15: 949-960.

29. Kwan LT, Reed BR, Eberling JL, et al. Effects of subcortical cerebral infarction on cortical glucose metabolism and cognitive function. Arch Neurol. 1999;56: 809-814.

30. Ziemann U, Muellbacher W, Hallett M, Cohen LG. Modulation of practicedependent plasticity in human motor cortex. Brain. 2001;124:1171-1181.

31. Levy LM, Ziemann U, Chen R, Cohen LG. Rapid modulation of GABA in sensorimotor cortex induced by acute deafferentation. Ann Neurol. 2002;52: 755-761.

32. Kaelin-Lang A, Luft AR, Sawaki L, Burstein AH, Sohn YH, Cohen LG. Modulation of human corticomotor excitability by somatosensory input. $J$ Physiol. 2002;540:623-633.

33. Park HJ, Kim JJ, Lee SK, et al. Corpus callosal connection mapping using cortical gray matter parcellation and DT-MR imaging. Hum Brain Mapp. 2008;29:503-516.

34. Paus T. Primate anterior cingulate cortex: where motor control, drive and cognition interface. Nat Rev Neurosci. 2001;2:417-424.

35. Shima K, Tanji J. Role for cingulate motor area cells in voluntary movement selection based on reward. Science. 1998;282:1335-1338.

36. Tanji J, Shima K. Role for supplementary motor area cells in planning several movements ahead. Nature. 1994;371:413-416.

37. Johansen-Berg H, Rushworth MF, Bogdanovic MD, Kischka U, Wimalaratna S, Matthews PM. The role of ipsilateral premotor cortex in hand movement after stroke. Proc Natl Acad Sci USA. 2002;99:14518-14523.

38. van Meer MP, van der Marel K, Wang K, et al. Recovery of sensorimotor function after experimental stroke correlates with restoration of resting-state interhemispheric functional connectivity. J Neurosci. 2010;30:3964-3972.

39. Chugani DC, Muzik O, Juhasz C, Janisse JJ, Ager J, Chugani HT. Postnatal maturation of human GABAA receptors measured with positron emission tomography. Ann Neurol. 2001;49:618-626. 